\title{
Theoretical Foundation for the Hume-Rothery Electron Concentration Rule for Structurally Complex Alloys
}

\author{
U. MizUtani ${ }^{a, *}$, H. SATO ${ }^{b}$, M. InUKAI ${ }^{c}$ And E.S. Zijlstra ${ }^{d}$ \\ ${ }^{a}$ Nagoya Industrial Science Research Institute, 1-13 Yotsuya-dori, Chikusa-ku, Nagoya, 464-0819, Japan \\ ${ }^{b}$ Aichi University of Education, Kariya-shi, Aichi, 448-8542, Japan \\ ${ }^{c}$ Nagoya Institute of Technology, Department of Frontier Materials, Gokiso-cho, Showa-ku, Nagoya 466-8555, Japan \\ ${ }^{d}$ Theoretical Physics, University of Kassel, 34132 Kassel, Germany
}

\begin{abstract}
An electron concentration parameter, expressed as the number of itinerant electrons per number of atoms $N$ in a structural unit cell of an alloy, $e / u c$, is a useful parameter that can be used in interpreting the origin of a band structure pseudogap often evident at the Fermi level for structurally complex metallic alloy phases. It can be expressed in terms of the interference condition $e / u c=\frac{\pi}{3}\left[|\boldsymbol{G}|_{\mathrm{c}}^{2}\right]^{3 / 2}$, where $|\boldsymbol{G}|_{\mathrm{c}}^{2}$ is the square of the critical reciprocal lattice vector associated with the specific set of lattice planes interfering with electrons at the Fermi level. This parameter is similar to the well-known Hume-Rothery electron concentration parameter $e / a$, which represents the number of electrons per atom and is linked with $e / u c$ through the relation $e / u c=N(e / a)$. We have demonstrated that certain complex metallic alloy structures appear to be stable at or near certain values of $e / a$. We show that the $e / a=1.60$ rule holds for the sub-group of gamma-brasses with space group $I \overline{4} 3 m$ and Pearson symbol $c I 52$, the $e / a=4.34$ rule for skutterudite compounds with $\operatorname{Im} \overline{3}$ and $c I 32$, the $e / a=2.74 \mathrm{rule}$ for $\mathrm{Al}_{6} \mathrm{TM}$ $(\mathrm{TM}=\mathrm{Mn}, \mathrm{Tc}, \mathrm{Re}, \mathrm{Fe}$, and Ru) compounds with $\mathrm{Cmcm}$ and $o C 28$, the $e / a=1.62$ rule for the sub-group of the Heusler compounds with $F m \overline{3} m$ and $c F 16$, and the $e / a=2.09$ rule for the sub-group of Zintl compounds $\mathrm{MX}_{1}$ $\left(\mathrm{M}=\mathrm{Li}\right.$ and $\mathrm{Na}, \mathrm{X}_{1}=\mathrm{Al}, \mathrm{Ga}$, In, and $\left.\mathrm{Tl}\right)$ with $F d \overline{3} m$ and $c F 32$. The $e / a$ rule holds in sub-groups of isostructural compounds, regardless of the degree of orbital hybridizations and the polarity involved.
\end{abstract}

DOI: 10.12693/APhysPolA.126.531

PACS: 71.20.Be, 71.20.Lp, 71.15.Mb

\section{Introduction}

Since the nominal valences for the pure noble metals and some number of polyvalent elements have been known a priori already in the 1920 s, one can relatively easily assign an average electrons per atom ratio $e / a$ for many noble metal alloy phases simply by taking a composition average of nominal valences of constituent elements across the phase diagram. Thanks to it, Hume-Rothery and his contemporaries could establish the so-called Hume-Rothery electron concentration rule for noble metal alloys in the 1930s [1]. More recently, Tsai et al. [2-4] could successfully synthesize a series of the Mackey icosahedral MI-type quasicrystals in $\mathrm{Al}-\mathrm{Cu}-\mathrm{TM}$ $(\mathrm{TM}=\mathrm{Fe}, \mathrm{Ru}$, and $\mathrm{Os})$ and $\mathrm{Al}-\mathrm{Pd}-\mathrm{TM}(\mathrm{TM}=\mathrm{Mn}$ and $\mathrm{Re}$ ) alloy systems by using the Hume-Rothery electron concentration rule as a guide. They claimed that they are stabilized at $e / a$ nearly equal to 1.8 , provided that the negative $e / a$ values which Raynor proposed in 1949 [5] are assigned to the transition metal (TM) elements. However, the assignment of negative $e / a$ values for TM elements has remained controversial. Indeed, it has become a central issue in metal physics why the $e / a$ -dependent phase stabilization mechanism or the Hume-Rothery stabilization mechanism works even for TM

*corresponding author; e-mail: uichiro@xa3.so-net.ne.jp containing compounds, where orbital hybridizations are significant. The effectiveness of the Hume-Rothery stabilization mechanism for a number of TM-bearing compounds including MI-type approximants to quasicrystals as well as the determination of reliable $e / a$ values for $3 d-$, $4 d$ - and $5 d$-TM elements has been recently established by making full use of the full-potential linearized augmented plane wave (FLAPW) Fourier method, as described below [6-11].

In the first-principles FLAPW electronic structure calculation method, the wave functions outside the muffin-tin (MT) spheres are expanded into plane waves over allowed reciprocal lattice vectors $\boldsymbol{G}$. By making full use of its formalism, Mizutani and coworkers [1, 6-11] have devised the FLAPW-Fourier method to extract FsBz (Fermi surface-Brillouin zone) interactions and to determine the $e / a$ value for elemental solids as well as intermetallic compounds, regardless of whether a TM element is involved, or not. It was emphasized that the number of electrons per unit cell, $e / u c$, in place of $e / a$, plays a key role in justifying the interference condition $\left(2 k_{\mathrm{F}}\right)^{2}=|\boldsymbol{G}|_{\mathrm{c}}^{2}$ or equivalently, $e / u c=\frac{\pi}{3}\left[|\boldsymbol{G}|_{\mathrm{c}}^{2}\right]^{3 / 2}$, where $\left(2 k_{\mathrm{F}}\right)^{2}$ is the square of the Fermi diameter and $|\boldsymbol{G}|_{\mathrm{c}}^{2}$ is the square of critical reciprocal lattice vector, which is defined such that the center of gravity energy in the energy dependence of the $|\boldsymbol{G}|^{2}$-wave at symmetry points of the Brillouin zone coincides with the Fermi level $E_{\mathrm{F}}$. Indeed, the $e / u c$ versus $|\boldsymbol{G}|_{\mathrm{c}}^{2}$ data for structurally complex metallic alloy (CMA) phases having a prominent pseudogap in 
the density of states (DOS) at $E_{\mathrm{F}}$ fall on a straight line with a slope of $3 / 2$ on a $\log -\log$ scale diagram [10]. This is taken as the validation of the Hume-Rothery stabilization mechanism, in which the pseudogap at $E_{\mathrm{F}}$ originates from the interference phenomenon mentioned above.

What about the role of $e / a$ ? The present work reports the FLAPW-Fourier analysis for a number of pseudogap CMAs to demonstrate that the $e / a=$ constant rule for many CMAs does hold not only for noble metal alloys but also for TM-bearing compounds. We thus provide a consistent theoretical basis towards a comprehensive understanding of the Hume-Rothery $e / a$-rule.

\section{Electronic structure calculations}

FLAPW band calculations have been performed by employing the WIEN2k program package [12]. Our in-house Fortran90 Program has been devised to carry out the FLAPW-Fourier analysis by using the "case.output1" file generated by running WIEN2k [1, 6-11]. Briefly, the FLAPW-Fourier spectrum was constructed by plotting the energy dependence of the plane wave specified by the square of reciprocal lattice vector $|\boldsymbol{G}|^{2}$ in units of $(2 \pi / a)^{2}$ for cubic systems or the sum of the Miller indices at selected symmetry points of the Brillouin zone. The $|\boldsymbol{G}|^{2}$-specified critical electronic state, i.e. $|\boldsymbol{G}|_{\mathrm{c}}^{2}$, can be extracted as the one, in which the center of gravity of its energy spectrum coincides with $E_{\mathrm{F}}$. The energy dispersion relation is also constructed for electrons outside the MT spheres. It is specifically called the Hume-Rothery plot, since it allows us to determine the square of the effective Fermi diameter $\left(2 k_{\mathrm{F}}\right)^{2}$ and the $e / a$ value for each intermetallic compound studied [1, 6-11].

\section{Results and discussions}

The family of gamma-brasses with space group $I \overline{4} 3 \mathrm{~m}$ (or $P \overline{4} 3 m$ ) (or) and the Pearson symbol $c I 52$ (or $c P 52$ ) has been divided into three subgroups, depending on the combination of atomic species of constituent elements: (1) noble metals and polyvalent elements, (2) TM elements and polyvalent elements, and (3) monovalent $\mathrm{Ag}$ and $\mathrm{Li}[1,11]$. To begin with, we direct our attention to the $\mathrm{Cu}_{5} \mathrm{Zn}_{8}$ and $\mathrm{Al}_{4} \mathrm{Cu}_{9}$ gamma-brasses in the subgroup 1 . They are characterized by a pseudogap at $E_{\mathrm{F}}$ and have already been proved to obey the Hume-Rothery stabilization mechanism with critical $|\boldsymbol{G}|^{2}=18$ and $e / a=1.60$ [1]. We confirmed that the $e / a=1.60$ rule is also justified for $\mathrm{Ag}_{5} \mathrm{Zn}_{8}$ and $\mathrm{In}_{4} \mathrm{Ag}_{9}$ gamma-brasses. In the case of the isostructural $\mathrm{Al}_{8} \mathrm{~V}_{5}$ gamma-brass in the subgroup 2, however, we revealed that a critical $|\boldsymbol{G}|^{2}$ is increased to 22 , appearing next to $|\boldsymbol{G}|^{2}=18$ at symmetry points $N$ of the bcc Brillouin zone, and that $e / a=2.19$ [11]. The pseudogap is masked by the $\mathrm{V} 3 d$ states and is no longer found at $E_{\mathrm{F}}$ in its total DOS. Thus, the $e / a=1.60 \pm 0.02$ rule holds in the subgroup 1 but not in the subgroups 2 and $3[1,11]$. The data for the subgroup 1 gamma-brasses obeying the $e / a=1.60$ rule are listed in Table.
TABLE

Numerical data relevant to the $e / a=$ constant rule for different families of CMAs.

\begin{tabular}{|c|c|c|c|c|c|}
\hline & System & $N$ & $|\boldsymbol{G}|^{2}$ & $\left(2 k_{\mathrm{F}}\right)^{2}$ & $e / a$ \\
\hline gamma-brasses & $\mathrm{Cu}_{5} \mathrm{Zn}_{8}$ & 52 & 18 & $18.5 \pm 0.1$ & \multirow{4}{*}{$1.60 \pm 0.02$} \\
\hline$I \overline{4} 3 m, c I 52$ & $\mathrm{Ag}_{5} \mathrm{Zn}_{8}$ & 52 & 18 & $18.5 \pm 0.1$ & \\
\hline$P \overline{4} 3 m, c P 52$ & $\mathrm{Al}_{4} \mathrm{Cu}_{9}$ & 52 & 18 & $18.5 \pm 0.1$ & \\
\hline in subgroup 1 & $\mathrm{In}_{4} \mathrm{Ag}_{9}$ & 52 & 18 & $18.5 \pm 0.1$ & \\
\hline \multirow{10}{*}{$\begin{array}{l}\text { skutterudites } \\
\text { Im } \overline{3}, c I 32\end{array}$} & $\mathrm{CoAs}_{3}$ & 32 & 26 & $25.2 \pm 0.2$ & \multirow{10}{*}{$4.34 \pm 0.20$} \\
\hline & $\mathrm{RhAs}_{3}$ & 32 & 26 & $26.3 \pm 0.2$ & \\
\hline & $\operatorname{Ir}_{\mathrm{As}_{3}}$ & 32 & 26 & $26.7 \pm 0.2$ & \\
\hline & $\mathrm{CoSb}_{3}$ & 32 & 26 & $26.5 \pm 0.2$ & \\
\hline & $\mathrm{RhSb}_{3}$ & 32 & 26 & $27.3 \pm 0.2$ & \\
\hline & $\mathrm{IrSb}_{3}$ & 32 & 26 & $27.8 \pm 0.2$ & \\
\hline & $\mathrm{NiP}_{3}$ & 32 & 26 & $25.1 \pm 0.2$ & \\
\hline & $\mathrm{CoP}_{3}$ & 32 & 26 & $25.0 \pm 0.2$ & \\
\hline & $\mathrm{RhP}_{3}$ & 32 & 26 & $25.9 \pm 0.2$ & \\
\hline & $\operatorname{IrP}_{3}$ & 32 & 26 & $26.7 \pm 0.2$ & \\
\hline \multirow{5}{*}{$\begin{array}{c}\mathrm{Al}_{6} \mathrm{TM} \\
\text { Cmcm, oC } 28\end{array}$} & $\mathrm{Al}_{6} \mathrm{Mn}$ & 28 & 17.4 & $17.3 \pm 0.3$ & \multirow{5}{*}{$2.74 \pm 0.05$} \\
\hline & $\mathrm{Al}_{6} \mathrm{Tc}$ & 28 & 17.5 & $17.5 \pm 0.3$ & \\
\hline & $\mathrm{Al}_{6} \mathrm{Re}$ & 28 & 17.5 & $17.6 \pm 0.3$ & \\
\hline & $\mathrm{Al}_{6} \mathrm{Fe}$ & 28 & 17.5 & $17.5 \pm 0.3$ & \\
\hline & $\mathrm{Al}_{6} \mathrm{Ru}$ & 28 & 17.5 & $17.5 \pm 0.3$ & \\
\hline Heusler & $\mathrm{Fe}_{2} \mathrm{VAl}$ & 16 & 8 & $8.1 \pm 0.3$ & \multirow{3}{*}{$1.62 \pm 0.20$} \\
\hline$F m \overline{3} m, c F 16$ & $\mathrm{Na}_{3} \mathrm{As}$ & 16 & 8 & $8.8 \pm 0.3$ & \\
\hline in subgroup 1 & $\mathrm{Li}_{3} \mathrm{Sb}$ & 16 & 8 & $8.7 \pm 0.2$ & \\
\hline in subgroup 2 & $\mathrm{AlTiAu}_{2}$ & 16 & 11 & $11.5 \pm 0.3$ & $2.55 \pm 0.10$ \\
\hline \multirow{5}{*}{$\begin{array}{c}\text { Zintl } \\
F d \overline{3} m, c F 32 \\
\text { in subgroup } 1\end{array}$} & $\mathrm{LiAl}$ & 32 & 16 & $16.3 \pm 0.2$ & \multirow{5}{*}{$2.09 \pm 0.06$} \\
\hline & $\mathrm{LiGa}$ & 32 & 16 & $16.0 \pm 0.2$ & \\
\hline & LiIn & 32 & 16 & $15.8 \pm 0.2$ & \\
\hline & NaIn & 32 & 16 & $16.0 \pm 0.2$ & \\
\hline & $\mathrm{NaTl}$ & 32 & 16 & $16.0 \pm 0.2$ & \\
\hline
\end{tabular}

We have extended our studies to the following families of compounds to examine whether the $e / a=$ constant rule holds: ten skutterudite compounds with space group $\operatorname{Im} \overline{3}$ and the Pearson symbol $c I 32$, five $\mathrm{Al}_{6} \mathrm{TM}$ $(\mathrm{TM}=\mathrm{Mn}, \mathrm{Tc}, \mathrm{Re}, \mathrm{Fe}$, and $\mathrm{Ru}$ ) compounds with $\mathrm{Cmcm}$ and $o C 28$, four Heusler compounds with $F m \overline{3} m$ and $c F 16$ and seven Zintl compounds $\mathrm{MX}_{1}(\mathrm{M}=\mathrm{Li}, \mathrm{Na}$ and $\mathrm{X}_{1}=\mathrm{Al}, \mathrm{Ga}$, In, and $\left.\mathrm{Tl}\right)$ and $\mathrm{MX}_{2}\left(\mathrm{M}=\mathrm{Li}\right.$ and $\mathrm{X}_{2}=\mathrm{Zn}$ and $\mathrm{Cd}$ ) with $F d \overline{3} m$ and $c F 32$. All structural information is available from the literature [13].

As the representative for the skutterudite compounds, the FLAPW-Fourier spectra at symmetry points $N$ along with the total DOS and the Hume-Rothery plot for the $\mathrm{CoAs}_{3}$ compound are depicted in Fig. 1a and b, respectively. A DOS pseudogap is clearly seen at the Fermi level. This is true for all skutterudite compounds studied. The Fourier coefficient $\sum\left|C_{\boldsymbol{k}+\boldsymbol{G}}\right|^{2}$ in each $|\boldsymbol{G}|^{2}$-specified wave remains finite at $E_{\mathrm{F}}$ over the range of $|\boldsymbol{G}|^{2}$ from 18 to 34 in $\mathrm{CoAs}_{3}$ (see the definition of the Fourier coefficient in Refs. [1, 6-11]). Nevertheless, we find that the center of gravity energy coincides with $E_{\mathrm{F}}$ at $|\boldsymbol{G}|^{2}=26$, as highlighted with red color. From the Hume-Rothery plot shown in Fig. 1b, we can determine both $\left(2 k_{\mathrm{F}}\right)^{2}$ and $e / a$ to be $25.2 \pm 0.2$ and 4.14 for $\mathrm{CoAs}_{3}$, respectively. All 
the relevant data for skutterudite compounds are summarized in Table. We see that all of them are characterized by a common critical $|\boldsymbol{G}|_{\mathrm{c}}^{2}=26$. Moreover, the values of $\left(2 k_{\mathrm{F}}\right)^{2}$ are distributed in the neighborhood of 26 , satisfying the common interference condition. Therefore, we conclude that the $e / a=4.34 \pm 0.20$ rule holds for all skutterudite compounds.

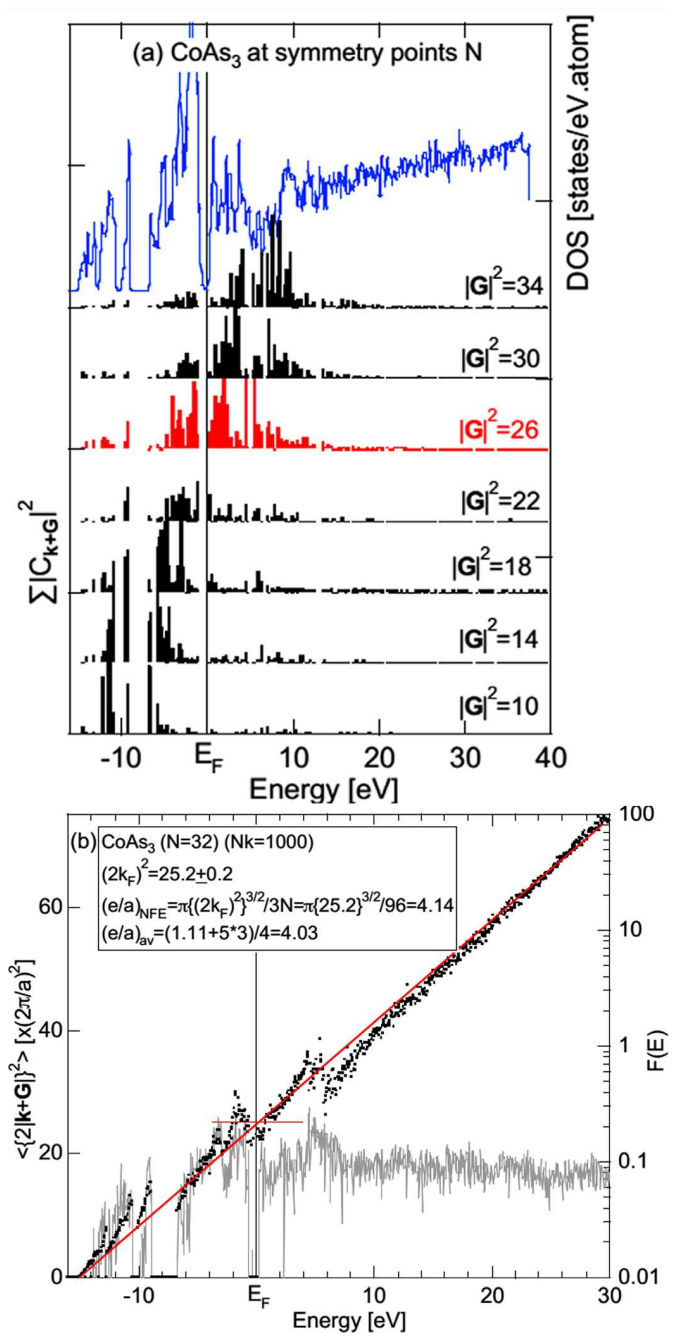

Fig. 1. FLAPW-Fourier spectra (a) at symmetry points $N$ for skutterudite compound $\mathrm{CoAs}_{3}$ along with its total DOS. The critical states are drawn in red. (b) Hume-Rothery plot for $\mathrm{CoAs}_{3} . \quad(e / a)_{\mathrm{av}}=4.03$ is obtained by taking a composition average of $(e / a)_{\mathrm{Co}}=$ $1.11[5]$ and $(e / a)_{\mathrm{As}}=5.0$.

The FLAPW-Fourier spectra and Hume-Rothery plot for the $\mathrm{Al}_{6} \mathrm{Mn}$ and $\mathrm{Al}_{6}$ Re compounds were already reported elsewhere [11]. As listed in Table for a series of $\mathrm{Al}_{6} \mathrm{TM}$ compounds, the critical $|\boldsymbol{G}|^{2}$ is distributed in the vicinity of 17.5 and the $e / a=2.74 \pm 0.05$ rule holds well. ${ }^{\dagger}$ It is emphasized that the $e / a$ value deduced for the re-

\footnotetext{
$\dagger$ In an orthorhombic crystal with lattice constants $a, b$, and $c$,
}

spective compounds can be well reproduced by taking a composition average of $e / a$ values of constituent elements $\mathrm{Al}$ and TM $[9,10]$.

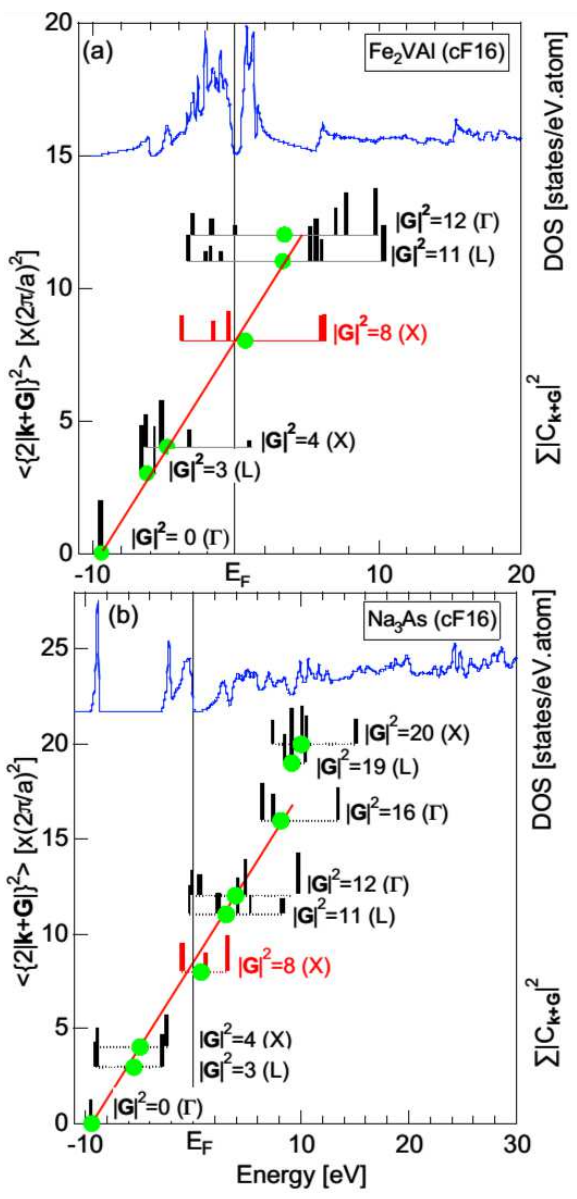

Fig. 2. FLAPW-Fourier spectra at symmetry points $N$ along with its total DOS for the Heusler-type compounds (a) $\mathrm{Fe}_{2} \mathrm{VAl}$ and (b) $\mathrm{Na}_{3} \mathrm{As}$. The center of gravity energy in each $|\boldsymbol{G}|^{2}$-specified wave is marked with green solid circles. The red line is drawn to pass through the center of gravity energy. The critical $|\boldsymbol{G}|^{2}$ state is highlighted with red color. Its value is commonly deduced to be 8 for both compounds.

Let us now turn our attention to the $e / a$-rule for the Heusler-type compounds with space group $F m \overline{3} m$ and Pearson symbol $c F 16$. According to Pearson's handbook [13], there are more than 350 compounds belonging to this family. Some of them possess either a true gap or a pseudogap at the Fermi level, while others do not, indicating the existence of diversified stabilization mechanisms in this family. In the present work, we focus only

$|\boldsymbol{G}|^{2}$ is expressed as $\left[\left(\frac{b c}{a^{2}}\right)^{2 / 3} h^{2}+\left(\frac{c a}{b^{2}}\right)^{2 / 3} k^{2}+\left(\frac{a b}{c^{2}}\right)^{2 / 3} l^{2}\right]$ in units of $\left(\frac{2 \pi}{V_{o}^{1 / 3}}\right)^{2}$, where $V_{0}=a b c$ is the volume of the unit cell and $(h k l)$ is the set of the Miller indices. Hence, the value of $|\boldsymbol{G}|^{2}$ becomes dependent on lattice constants. 
on the gap systems, since its origin can be discussed in terms of the interference condition or Hume-Rothery stabilization mechanism.

Figure $2 \mathrm{a}$ and $\mathrm{b}$ shows the FLAPW-Fourier spectra at symmetry points $\Gamma, L$, and $X$ along with the total DOS for the compounds $\mathrm{Fe}_{2} \mathrm{VAl}$ and $\mathrm{Na}_{3} \mathrm{As}$, respectively. The center of gravity energy for each $|\boldsymbol{G}|^{2}$-specified wave is marked with green solid circles. It is clear that $|\boldsymbol{G}|^{2}=8$ can be commonly extracted as being critical in both compounds. As is listed in Table, $\mathrm{Fe}_{2} \mathrm{VAl}, \mathrm{Na}_{3} \mathrm{As}$, and $\mathrm{Li}_{3} \mathrm{Sb}$ compounds are proved to possess common electronic parameters: critical $|\boldsymbol{G}|^{2}=8$ and $e / a=1.62 \pm 0.20$. The pseudogap compound $\mathrm{AlTiAu}_{2}$ belongs to this family having the next higher critical $|\boldsymbol{G}|^{2}$, that is, 11 . Its $e / a$ value is stepwise increased to $2.55 \pm 0.10$. Thus, we can say that $\mathrm{Fe}_{2} \mathrm{VAl}, \mathrm{Na}_{3} \mathrm{As}$, and $\mathrm{Li}_{3} \mathrm{Sb}$ compounds belong to the subgroup 1 and that $\mathrm{AlTiAu}_{2}$ to the subgroup 2 in the family of the Heusler-type compounds.

Finally, we focus on the Zintl compounds $\mathrm{MX}_{1}(\mathrm{M}=\mathrm{Li}$ and $\mathrm{Na}, \mathrm{X}_{1}=\mathrm{Al}, \mathrm{Ga}, \mathrm{In}$, and $\left.\mathrm{Tl}\right)$ and $\mathrm{MX}_{2}(\mathrm{M}=\mathrm{Li}$ and $\mathrm{X}_{2}=\mathrm{Zn}$ and $\mathrm{Cd}$ ) with space group $F d \overline{3} m$ and Pearson symbol $c F 32$. All of them are known to exhibit a strong ionic bonding tendency among constituent elements. For example, a complete charge transfer occurs from $\mathrm{Na}$ to $\mathrm{Tl}$ in $\mathrm{NaTl}$ and results in ionic bonding $\mathrm{Na}^{+} \mathrm{Tl}^{-}$. As a result, $\left[\mathrm{Tl}^{-}\right]$behaves as if it has 4 valence electrons and forms a covalent diamond structure together with $\mathrm{Na}^{+}$cations stuffing the lattice. It is highly interesting to study whether the $e / a$ rule holds in such polar compounds.

As summarized in Table, a critical $|\boldsymbol{G}|^{2}$ is commonly deduced to be 16 and values of $\left(2 k_{\mathrm{F}}\right)^{2}$ are distributed in the vicinity of 16 for the $\mathrm{MX}_{1}$ compounds. The $e / a=2.09 \pm 0.06$ rule is revealed in the subgroup 1 of the Zintl compounds consisting of alkali metals and trivalent elements. The nominal $e / a$ value calculated from a composition average of valences of constituent elements is obviously two. A critical $|\boldsymbol{G}|^{2}$ is stepwise decreased to 12 but a pseudogap is no longer present at the Fermi level in the subgroup 2 of the Zintl compounds $\mathrm{MX}_{2}$. Thus, we can say that the $e / a=2.09 \pm 0.06$ rule holds in the subgroup 1 of the Zintl compounds. It is more important to emphasize that the $e / a=$ constant rule is justified, regardless of the degree of ionicity and polarity.

\section{Summary}

We could lay down a theoretical foundation for the $e / a$ rule in the family of isostructural compounds using the following conditions as a guide. First, the isostructural compounds are defined as those belonging to a given space group and the Pearson symbol. The number of atoms per unit cell is thus the same. In the case of I-type compounds with bcc lattice, for example, successively appearing $|\boldsymbol{G}|^{2}$ s at symmetry points $N$ of its Brillouin zone are common: 2, 6, 10, 14, 18, 22, 26 , $30,34 \ldots$ corresponding to $\{110\},\{211\},\{310\},\{321\}$, $\{330\}+\{411\}$ etc. Hence, critical $|\boldsymbol{G}|_{\mathrm{c}}^{2}$ must be selected from such discrete sets of $|\boldsymbol{G}|^{2}$ series. Moreover, the interference condition $\left(2 k_{\mathrm{F}}\right)^{2}=|\boldsymbol{G}|_{\mathrm{c}}^{2}$ is imposed for a pseudogap system. As a result, compounds obeying the $e / a$ rule can be extracted as a subgroup of isostructural compounds, where a common critical $|\boldsymbol{G}|_{\mathrm{c}}^{2}$ is assigned in the presence of the interference condition, thereby guaranteeing the possession of identical $\left(2 k_{\mathrm{F}}\right)^{2}$ and $e / a$ value. It may be worthwhile noting that the common $e / a$ does not exist between the $\mathrm{Na}-\mathrm{Au}-\mathrm{Ga}$ and $\mathrm{Al}-\mathrm{Mg}-\mathrm{Zn}$ isostructural compounds [14] but that their respective $e / a$ values are determined through different critical $|\boldsymbol{G}|_{\mathrm{c}}^{2}$ selected from the same series of allowed $|\boldsymbol{G}|^{2}$ s. Thus, they belong to different subgroups in the family of isostructural compounds, as has been emphasized in the present article.

\section{Acknowledgments}

One of the authors (U.M.) expresses his deep thanks to Emeritus Professor T.B. Massalski, Carnegie-Mellon University, Pittsburgh, for fruitful discussions and providing valuable comments to the manuscript. He is also grateful for the financial support of the Grant-in-Aid for Scientific Research (contract No. 23560793) from the Japan Society for the Promotion of Science.

\section{References}

[1] U. Mizutani, Hume-Rothery Rules for Structurally Complex Alloy Phases, CRC Press Taylor \&Francis Group, Boca Raton 2010.

[2] A.P. Tsai, A. Inoue, T. Masumoto, Jpn. J. Appl. Phys. 27, L1587 (1988).

[3] A.P. Tsai, A. Inoue, Y. Yokoyama, T. Masumoto, Mater. Trans. Jpn. Inst. Met. 31, 98 (1990).

[4] Y. Yokoyama, A.P. Tsai, A. Inoue, T. Masumoto, H.S. Chen, Mater. Trans. Jpn. Inst. Met. 32, 421 (1991).

[5] G.V. Raynor, Prog. Met. Phys. 1, 1 (1949).

[6] M. Inukai, E.S. Zijlstra, H. Sato, U. Mizutani, Philos. Mag. 91, 4247 (2011).

[7] U. Mizutani, M. Inukai, H. Sato, E.S. Zijlstra, Philos. Mag. 92, 1691 (2012).

[8] U. Mizutani, M. Inukai, H. Sato, E.S. Zijlstra, Chem. Soc. Rev. 41, 6799 (2012).

[9] H. Sato, M. Inukai, E.S. Zijlstra, U. Mizutani, Philos. Mag. 93, 3029 (2013).

[10] U. Mizutani, H. Sato, M. Inukai, E.S. Zijlstra, Philos. Mag. 93, 3353 (2013).

[11] U. Mizutani, M. Inukai, H. Sato, E.S. Zijlstra, in: Physical Metallurgy 5th ed., Eds. K. Hono, D. Laughlin, Elsevier, Amsterdam 2013, p. 1.02.

[12] P. Blaha, K. Schwarz, G. Madsen, D. Kvasnicka, J. Luitz, WIEN2k, (last accessed on August 30, 2013).

[13] P. Villars, Pearson's Handbook, Crystallographic Data, ASM, Materials Park, OH 1997.

[14] U. Mizutani, H. Sato, M. Inukai, E.S. Zijlstra, Q. Lin, J.D. Corbett, G.J. Miller, Acta Phys. Pol. A 126, 535 (2014). 